\title{
Empirical Doppler Characterization of Signals Scattered by Wind Turbines in the UHF Band under Near Field Condition
}

\author{
Itziar Angulo, ${ }^{1}$ Jon Montalbán, ${ }^{1}$ Josune Cañizo, ${ }^{1}$ Yiyan $\mathrm{Wu},{ }^{2}$ David de la Vega, ${ }^{1}$ \\ David Guerra, ${ }^{1}$ Pablo Angueira, ${ }^{1}$ and Amaia Arrinda ${ }^{1}$ \\ ${ }^{1}$ Department of Communications Engineering, University of the Basque Country (UPV/EHU), Alda. Urquijo s/n, \\ 48013 Bilbao, Spain \\ ${ }^{2}$ Communications Research Centre, Ottawa, ON, Canada K2H 852
}

Correspondence should be addressed to Itziar Angulo; itziar.angulo@ehu.es

Received 29 October 2012; Accepted 21 January 2013

Academic Editor: Lars Norin

Copyright (C) 2013 Itziar Angulo et al. This is an open access article distributed under the Creative Commons Attribution License, which permits unrestricted use, distribution, and reproduction in any medium, provided the original work is properly cited.

Time variability of the scattering signals from wind turbines may lead to degradation problems on the communication systems provided in the UHF band, especially under near field condition. In order to analyze the variability due to the rotation of the blades, this paper characterizes empirical Doppler spectra obtained from real samples of signals scattered by wind turbines with rotating blades under near field condition. A new Doppler spectrum model is proposed to fit the spectral characteristics of these signals, providing notable goodness of fit. Finally, the effect of this kind of time variability on the degradation of OFDM signals is studied.

\section{Introduction}

Signal variability caused by blade rotation is a critical issue when we are dealing with potential degradations on communications services due to wind farms. For example, in the case of the UHF band, the potential increment on the minimum requirements for quasi-error-free reception of the DVB-T service is directly related to the channel time variability [1].

However, the classical scattering models used for the UHF band are based on worst case assumptions with respect to blade position and do not characterize time variability due to blade rotation [2]. The characterization of the Doppler spectra due to blade motion would be especially interesting for future implementation on channel models to characterize signal propagation in presence of a wind farm in this band.

The study presented in this paper is based on real scattering data obtained from a measurement campaign in the surroundings of a wind farm. Based on these data, the Doppler spectra are estimated. Then a general methodology to characterize these empirical Doppler power spectral densities (PSDs) is proposed, and finally an in-depth analysis of the effect of this type of variability on OFDM signals is carried out.
This paper is organized as follows. Section 2 introduces Doppler PSD estimation, describing the empirical source data and some existing methods for PSD estimation. Next, Section 3 presents the general methodology proposed to characterize the obtained Doppler PSDs, whose necessity is found after studying the classical Doppler spectrum models. Section 4 provides an analysis of the effect of the time variability due to blade rotation on OFDM signals. Finally, the main conclusions of the paper are gathered in Section 5.

\section{Doppler Power Spectral Density Estimation}

2.1. Empirical Source Data. The following analysis is based on UHF signals scattered by a wind farm which were recorded and postprocessed in order to detect the contributions of each individual wind turbine. Detailed descriptions of the field trials carried out to collect these signals and the methodology to obtain the scattering signals of each wind turbine from the recorded data can be found in previous references from the authors [1-4].

More precisely, the source data set is composed of 328 complex scattered signals lasting 10 seconds each, and corresponding to four different wind turbines that scattered signals 
from two nearby located television transmitters. Sampling period is given by the DVB-T symbol duration time, $T_{s}=$ $1120 \mu \mathrm{s}$, in such a way that the estimated PSDs will be obtained for a Doppler frequency range of $\pm 446.43 \mathrm{~Hz}$. This value is higher than the maximum Doppler shift $f_{d}$ of the case under study, which corresponds to monostatic reception condition and depends on the maximum rotation rate $w_{\max }$, blade length $l$ and transmission wavelength $\lambda$, according to

$$
f_{d}=\frac{2 w_{\max } l}{\lambda} .
$$

By way of illustration, Figure 1 shows an example of a signal scattered by a wind turbine as their blades rotate. A periodic variation which corresponds to $1 / 3$ of the rotation period of the rotor can be observed.

The distances between the transmitter sites and these four wind turbines are in a range from 250 to 950 meters. In the context of signal scattering and for practical measurement situations, near field effects occur when the target is not illuminated by a plane wave, and thus, the phase of the incident wave at the center of the target is different from the phase at its extremes. A widely accepted requirement is to limit the phase deviation to be less than $22.5^{\circ}$, obtaining the condition of far field distance for signal scattering $R_{0}$ as a function of the lateral dimension of the object $D$, according to [5]

$$
R_{0}=\frac{2 D^{2}}{\lambda}
$$

Taking into account the wind turbine dimensions (a mast of $55 \mathrm{~m}$ height and rotor of $52 \mathrm{~m}$ diameter), the scattering signals clearly correspond to near field condition. Although the spectral characteristics of the scattered signals will differ from those of the signals scattered under near field condition [6], it is precisely under near field condition that more severe degradations can occur for the telecommunication services provided in the UHF band [1], so these situations are of particular interest.

2.2. Methods for Power Spectral Density Estimation. Theoretically, it is enough to apply a discrete Fourier transform to the complex scattered signals along the observation time and take the squared absolute value of the result to obtain the spectral power distribution for the different Doppler frequencies. Nevertheless, as finite time signals are used, the effect is equivalent to multiplying the input signal $x(n)$ by a rectangular window [7].

Since the spectral characteristic of the windowing function $W(f)$ is relatively narrow with respect to the input signal spectrum $X(f)$, the convolution of $W(f)$ and $X(f)$ makes the energy of $X(f)$ to be distributed into secondary lobes where the original signal spectrum was null. This energy leaking can be reduced by using windowing functions featuring secondary lobes of lower amplitude. However, these windowing functions make the spectral characteristics of $X(f)$ to be wider due to the wider main lobe of the window spectrum, reducing frequency resolution [7-9].

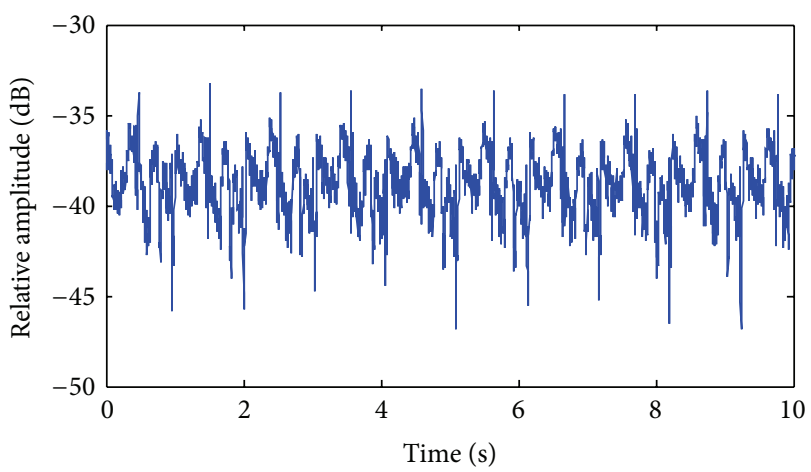

FIGURE 1: Example of the periodic time variability of the scattering signals as blades rotate.

In order to lessen these effects on the input signal characteristics, there are multiple power spectral estimation methods, normally classified as parametric, nonparametric, and subspace $[7,10,11]$. For the presented study, nonparametric methods are analyzed, as they make no assumptions about how the data were generated, and the input signal is directly used to obtain the power spectral density (PSD).

Five nonparametric methods are described in the literature: periodogram [12], modified periodogram, Bartlett's method [13], Blackman-Tukey, and Welch's method [14]. In fact, all of them are variations with respect to the classic periodogram method proposed by Schuster [12].

The periodogram is defined as [7]

$$
\widehat{S}_{x x}(k)=\frac{1}{N}\left|\sum_{n=0}^{N-1} x(n) \exp \left(-\frac{2 \pi j k n}{n}\right)\right|^{2} .
$$

The periodogram is asymptotically unbiased, but it is not a consistent estimate of the true PSD because the variance of the periodogram does not tend to zero as data length $N$ increases.

The modified periodogram uses a nonrectangular window to reduce the amplitude of the secondary lobes, but it does not solve the problem of the inconsistence.

In order to reduce the variance of the estimator, the Bartlett's method [13] divides the original signal into $K$ nonoverlapping segments of length $L=N / K$. Then the periodogram is calculated for each of these segments, and the obtained results are averaged. This way, the variance is reduced by a factor $K$, but the spectral resolution is reduced by the same factor [7].

Finally, Welch's method proposed two modifications to the Bartlett's method to reduce the variance without affecting the spectral resolution in the same proportion. First, the temporal segments are overlapped, and second, different windowing functions are applied to the data segments prior to computing the periodogram. The combined use of short data records and nonrectangular windows results in reduced resolution of the estimator, so that a tradeoff between variance reduction and resolution should be taken into account. 
The periodic nature of the signals scattered by the wind turbines due to blade rotation makes Welch's method especially suitable for the Doppler PSD estimation. In fact, the length $L$ of the data segments is adapted to coincide with the variation period of each signal. Apart from this, a 50\% overlap is applied and Hamming windowing for each segment is used [14].

\section{Doppler Spectra Characterization}

Although some references can be found in the literature about the spectral characteristics of the signals scattered by wind turbines [15-18], these studies are mainly based on the radar frequencies, and they do not aim at providing fitting models to represent the obtained spectrograms. Moreover, they deal with monostatic Doppler, whereas this paper is focused on bistatic Doppler.

3.1. State of the Art of Doppler Spectrum Models. Prior to the characterization of the obtained Doppler PSDs, the existing Doppler spectrum models should be studied in order to determine their empirical or theoretical basis and their application conditions.

3.1.1. Jakes Doppler Spectrum or Classical Model. The classical Jakes model is commonly used to characterize propagation for a mobile receiver. It is based on the following assumptions $[19,20]$.

(i) Radio waves propagate horizontally, in a two-dimensional plane, and the receiver is located in the centre of an isotropic scattering area.

(ii) The angles of arrival of the waves arriving the receiving antenna are uniformly distributed in the interval $[-\pi, \pi)$.

(iii) The radiation pattern of the receiving antenna is omnidirectional.

From these assumptions, the Jakes power spectral density is obtained:

$$
S_{j}(f)=\frac{1}{\pi f_{d} \sqrt{1-\left(f / f_{d}\right)^{2}}}, \quad|f| \leq f_{d},
$$

where $f_{d}$ is the maximum Doppler shift due to the receiver speed.

3.1.2. Flat Doppler Spectrum. In a three-dimensional scenario with isotropic scattering, being the angles of arrival of the waves uniformly distributed for both the horizontal and the vertical plane, the resulting Doppler spectrum is flat, and its PSD is given by (5) [21]

$$
S_{f}(f)=\frac{1}{2 f_{d}}, \quad|f| \leq f_{d} .
$$

3.1.3. Gaussian Doppler Spectrum. The Gaussian Doppler spectrum is used to model the multipath components with long delays in UHF communications, in the HF channel and aeronautical communications in the VHF band [22]. The PSD of the Gaussian Doppler spectrum is given by (6)

$$
S_{g}(f)=\frac{1}{\sqrt{2 \pi \sigma_{g}^{2}}} \exp \left(-\frac{f^{2}}{2 \sigma_{g}^{2}}\right) \text {. }
$$

3.1.4. Rounded Doppler Spectrum. The Rounded Doppler spectrum is proposed in [23] as an approximation to measured PSDs for the scattered component in a fixed wireless channel at $2.5 \mathrm{GHz}$. The PSD of the Rounded Doppler spectrum is given by (7)

$$
S_{r}(f)=C_{r}\left[a_{0}+a_{2}\left(\frac{f}{f_{d}}\right)^{2}+a_{4}\left(\frac{f}{f_{d}}\right)^{4}\right], \quad|f| \leq f_{d}
$$

where the normalization factor $C_{r}$ is given by (8)

$$
C_{r}=\frac{1}{2 f_{d}\left[a_{0}+a_{2} / 3+a_{4} / 5\right]} \text {. }
$$

Taking into account that the modeled channel features a lineof sight fixed component, a Dirac delta is included in the PSD for $f=0 \mathrm{~Hz}[23]$.

3.2. Characterization of Doppler PSDs due to Blade Rotation. As previously observed, the basic parameter to characterize Doppler spectra is the maximum Doppler frequency $f_{d}$. For a wind turbine in motion, the maximum theoretical Doppler frequency shift is obtained from the blade length, the transmission wavelength, and the maximum rotation rate of the wind turbine. However, this maximum Doppler frequency shift only corresponds to a monostatic situation.

For a general bistatic case, the maximum observable frequency depends not only on the blades characteristics and the maximum rotation rate but also on the relative location transmitter-wind turbine-receiver and the orientation of the rotor, according to [24]

$$
f_{B}=\frac{2 w l}{\lambda} \cos \delta \cos \left(\frac{\beta}{2}\right)
$$

where $w$ stands for the rotation rate, $l$ is the blade length, $\lambda$ is the wavelength, $\beta$ is the bistatic angle (transmitter-wind turbine-receiver), and $\delta$ is the angle defined by the rotation plane of the rotor with respect to the bistatic bisector, as observed in Figure 2.

Therefore, for a certain reception location and considering all the possible rotor orientations, the maximum bistatic Doppler frequency is given by

$$
f_{B_{-} \max }=\frac{2 w_{\max } l}{\lambda} \cos \left(\frac{\beta}{2}\right) .
$$

Bearing this in mind, two examples of estimated Doppler PSDs are shown in Figure 3. The common characteristic to all of the obtained PSDs is the presence of a main component at $0 \mathrm{~Hz}$, and decreasing spectral densities for the higher and 


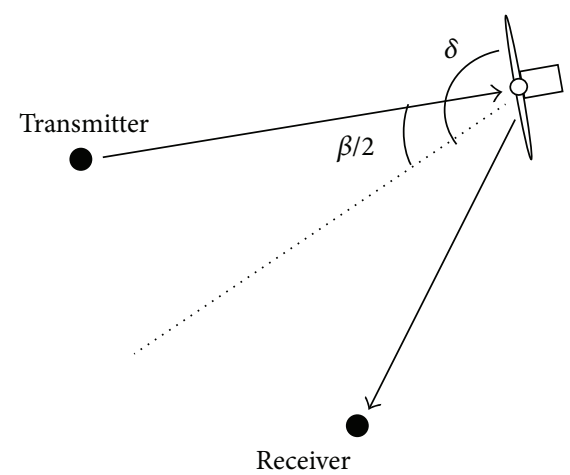

FIGURE 2: Geometry for bistatic Doppler in the horizontal plane.

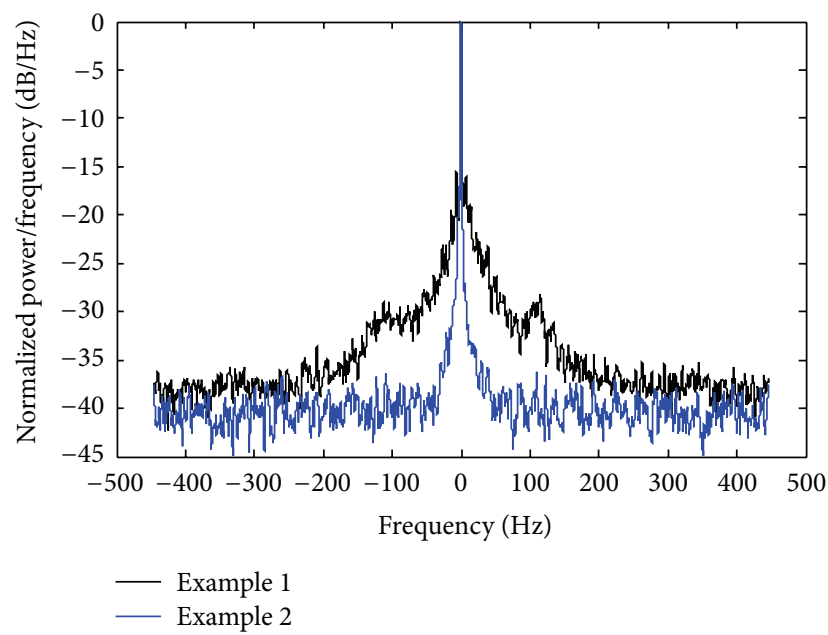

FIGURE 3: Examples of estimated PSDs corresponding to time variability of the scattering signals as blades rotate.

lower frequencies. All the PSDs are normalized with respect to the $0 \mathrm{~Hz}$ component prior to characterization $[25,26]$.

The spectral characteristics of the estimated PSDs are similar to those of fixed wireless communications where transmitter and receiver are static and the environment is responsible for the time variability of the channel [23, 27-29]. On the other hand, the presence of this main component for $f=0 \mathrm{~Hz}$ coincides with the conclusions obtained from physical optics simulations of the radar cross section of a wind turbine. According to [30], the main static component is due to the signal scattered by the mast, which can be significantly higher than the signal scattered by the blades. Regarding the characteristics of the Doppler spectra corresponding to the scattering from the blades, it is difficult to relate the spectrum shape to the geometry of the blades, due to their complex aerodynamic design. Moreover, the scattering from the blades is strongly dependent on the relative position transmitterwind turbine-receiver and the rotor orientation with respect to the wind direction.

These spectral characteristics do not match with the Doppler models presented in the previous section. Therefore, a new model is proposed, which is based on a PSD characterized by a Dirac delta for $f=0 \mathrm{~Hz}$ and exponential fittings for both the negative and positive frequencies [23, 27-29]. This way, the estimated Doppler PSDs are fitted in $\mathrm{dB} / \mathrm{Hz}$, after normalization with respect to the main component for $f=0 \mathrm{~Hz}$, according to

$$
S_{\delta}(f)= \begin{cases}a \exp (b f)-c & f<0 \\ \delta(f) & f=0 \\ d \exp (-e f)-g & f>0,\end{cases}
$$

where $a, b, c, d, e$, and $g$ are positive constants, $f$ stands for the Doppler frequency $(\mathrm{Hz})$ and $\delta(0)=0 \mathrm{~dB} / \mathrm{Hz} . b$ and $e$ represent the exponential decay (wider spectral characteristics for lower values and vice versa), $c$ and $g$ are related to the asymptotic values for infinite negative and positive frequencies, respectively, and $a$ and $d$ account for the relative value of the curve for the $y$-axis with respect to the asymptotic values for infinite frequencies given by $c$ and $g$.

For a correct characterization, the estimated Doppler PSDs should be frequency limited to avoid the noise floor being confused with the Doppler spectrum of the received signal. As previously commented, the maximum Doppler shift for a certain reception location depends on the orientation of the rotor against the wind and the rotational speed at that very moment, which is constantly varying and quite difficult to accurately calculate for each specific measurement.

Therefore, after analyzing the exponential decrease of the estimated PSDs and their relation to the noise threshold, the frequency limits of each PSD are calculated according to (12), assuming that the noise threshold is reached for the $99 \%$ of the asymptotic values given by $c$ and $g$, respectively:

$$
\begin{gathered}
S_{\delta}\left(f_{\min }\right)=0.99 c, \\
S_{\delta}\left(f_{\max }\right)=0.99 g .
\end{gathered}
$$

That is to say, using a nonlinear least squares method, the expression in (11) is fitted to the empirical PSDs. As a function of the obtained fitting parameters, the minimum and maximum frequency values $f_{\min }$ and $f_{\max }$ are calculated.

The goodness of fit of this Doppler model is evaluated by means of the coefficient of determination $R^{2}$, which represents the proportion of variability in a data set that is accounted for by a statistical model [31]. Values closer to 1 mean better goodness of fit. The mean $R^{2}$ value for the set of measurements under analysis is equal to 0.8. Apart from the goodness of fit of the proposed model, it can be observed that this methodology provides a systematical, adaptable, and efficient method to establish the frequency limits of the obtained PSDs (see Figures 4 and 5).

Finally, it should be mentioned that for the estimated PSDs featuring wider spectral characteristics, the proposed method can make the calculated frequency limits $f_{\min }$ and $f_{\max }$ to be out of the theoretical margin $-f_{B_{-} \max } \leq f \leq$ $f_{B_{-} \max }$. In this case, the frequency limits are set to $f_{\min }=$ $-f_{B_{-} \max }$ or $f_{\max }=f_{B_{-} \max }$, as corresponding. It should be noted that this is only the case of 4 out of 328 available signal samples.

Once the frequency limits are established and the noise influence is avoided, a second characterization of the Doppler 


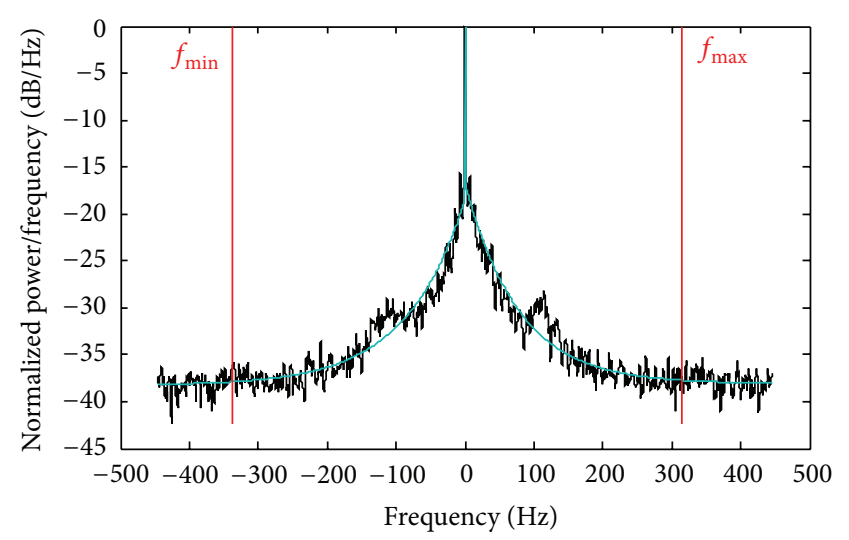

FIgURE 4: Frequency limits for Example 1.

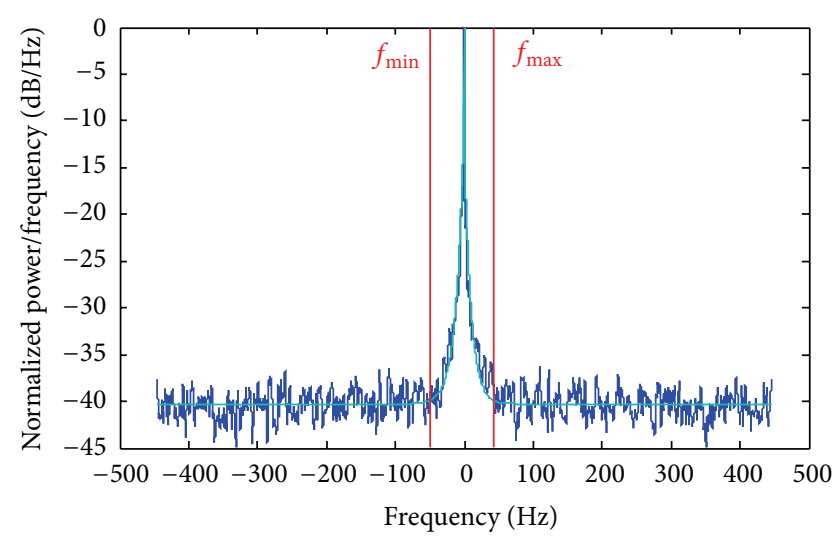

FIGURE 5: Frequency limits for Example 2.

spectra limited by $f_{\min } \leq f \leq f_{\max }$ is carried out using the model $S_{\delta}(f)$, according to (13). Applying this process to the whole set of empirical data, the final mean value of $R^{2}$ is equal to 0.87 , with a standard deviation of 0.09 , which demonstrates the goodness of fit of the proposed model. Consider

$$
S_{\delta}(f)= \begin{cases}a \exp (b f)-c & f_{\min } \leq f<0 \\ \delta(f) & f=0 \\ d \exp (-e f)-g & 0<f \leq f_{\max }\end{cases}
$$

\section{Behavior of the Obtained PSDs on OFDM Signals}

The objective of evaluating the influence of the obtained Doppler spectra on OFDM signals is twofold. First, an analysis of the most influential characteristics of the theoretical PSDs on this potential quality degradation is carried out. Second, it is checked whether the estimated spectra and their corresponding fitting expressions behave in a similar manner in terms of quality degradation.

4.1. Simulation of BER versus SNR Curves. The BER versus SNR curves are obtained using an OFDM software implementation that includes the simulation of configurable
Tapped Delay-Line (TDL) channel models [32]. This system deals with uncoded symbol reception. However, this condition does not affect the conclusions of the comparative study of the different estimated and fitted PSDs.

The selected OFDM configuration is close to the DVB-T configuration used in Spain [33]. A 64-QAM modulation is used, in an $8 \mathrm{~K}$ mode with a guard interval equal to a quarter of the symbol length, using a total bandwidth of $10 \mathrm{MHz}$ in the $800 \mathrm{MHz}$ band.

A TDL channel model is composed of a series of paths, each of them with a certain time delay and mean amplitude, and a Doppler spectrum to account for the channel time variability $[19,34,35]$. Therefore, a representative set of delays and their corresponding gains from a real situation from the measurement campaign has been selected in order to analyze the different Doppler spectra.

Regarding the Doppler spectra, it is necessary to select, from the 328 obtained samples and their corresponding exponential fittings, a significant number of examples for the simulation. To do so, after an analysis of the typologies of the estimated PSDs, the spectral width and the asymmetry in the power spectral distribution around $0 \mathrm{~Hz}$ are considered to be the main characteristics to be studied. Therefore, the following parameters are defined: $\Delta f$ is used as an indicator of the spectral width, according to (14); $\gamma$ represents the asymmetry of the PSD, according to (15); as previously commented, $R^{2}$ is a measure of the goodness of fit. Consider

$$
\begin{gathered}
\Delta f=f_{\max }-f_{\text {min }}, \\
\gamma=\frac{\left|f_{\text {max }}-\right| f_{\text {min }}||}{\Delta f} .
\end{gathered}
$$

Based on these parameters, 24 representative spectrum examples featuring different spectral widths and degrees of symmetry are selected in order to observe their influence on a potential degradation of the OFDM services, and different values of $R^{2}$ to evaluate the behavior of the exponential fittings against the empirical Doppler spectra. It should be noted that in the simulations, both the empirical Doppler spectra and the exponential fittings are limited in frequency as a function of the calculated values of $f_{\min }$ and $f_{\max }$. A representative set of 7 of these 24 Doppler spectra, which are used to exemplify the following analysis, is included in Appendix. In addition to this, the fitting parameters of these examples according to (13) are included in Table 4 (Appendix).

4.2. Analysis of the Results. In order to evaluate the behavior of the different spectra, the SNR values for a BER equal to $2 \cdot 10^{-4}$ (Quasi-Error-Free threshold for DVB-T [33]) are compared between the static curve (no Doppler spectrum used for the different paths) and the time-varying cases (empirical or fitted PSDs used to characterize channel variability in the different paths).

The results are classified according to the different factors that are going to be analyzed: the effect of the spectral width and the asymmetry of the Doppler PSD, and the goodness of fit of the proposed exponential fitting. 
TABLE 1: Parameters of the representative Doppler spectra.

\begin{tabular}{|c|c|c|c|c|c|c|}
\hline Example & $\Delta f(\mathrm{~Hz})$ & $\gamma$ & mean $\{c, g\}$ & $\Delta \mathrm{SNR}_{\text {est }}(\mathrm{dB})$ & $R^{2}$ & $\Delta \mathrm{SNR}_{\exp }(\mathrm{dB})$ \\
\hline A & 651.11 & 0.04 & 38.06 & 10.02 & 0.89 & 1.57 \\
\hline B & 821.82 & 0.00 & 49.16 & 7.46 & 0.70 & 6.39 \\
\hline $\mathrm{C}$ & 468.29 & 0.09 & 29.96 & 5.18 & 0.93 & 0.22 \\
\hline $\mathrm{D}$ & 241.12 & 0.31 & 40.32 & 0.53 & 0.86 & 0.13 \\
\hline $\mathrm{E}$ & 256.45 & 0.10 & 40.66 & 0.55 & 0.82 & 0.16 \\
\hline $\mathrm{F}$ & 473.87 & 0.03 & 44.60 & 1.13 & 0.90 & 0.06 \\
\hline G & 247.96 & 0.06 & 40.47 & 0.61 & 0.88 & 0.02 \\
\hline
\end{tabular}

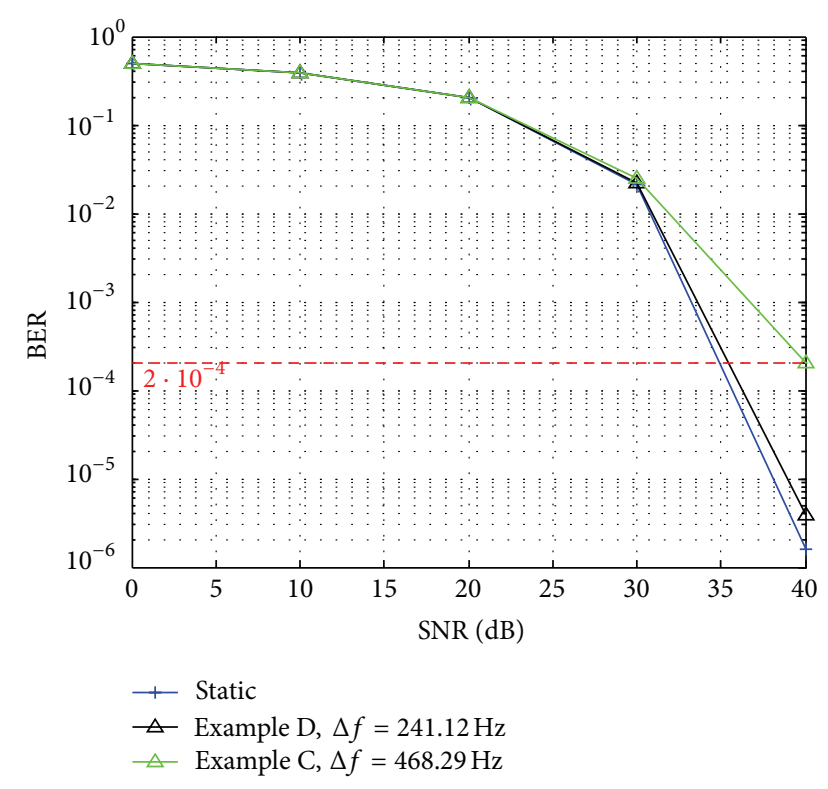

FIGURE 6: BER versus SNR curves for the static case and the empirical Doppler spectra of Examples C and D.

4.2.1. Effect of the Spectral Width. Figure 6 shows the curve corresponding to the static case (no Doppler spectrum used) depicted in blue, and two curves corresponding to the empirical Doppler spectra of Examples D and C, with increasing spectral widths $\left(\Delta f_{\mathrm{D}}=241.12 \mathrm{~Hz}\right.$ and $\Delta f_{\mathrm{C}}=468.29 \mathrm{~Hz}$, resp. See Table 4 and Figures 11 and 12 in Appendix). It can be observed that the difference in SNR between the static curve and the curve corresponding to the wider Doppler spectrum is significantly greater than the other case.

Taken the static channel as a reference, Figure 7 shows the SNR values corresponding to a BER value of $2 \cdot 10^{-4}$ for the selected PSDs, $\triangle \mathrm{SNR}_{\text {est }}$, as a function of their spectral width, $\Delta f$. It can be observed that the differences with respect to the static case $\Delta \mathrm{SNR}_{\text {est }}$ are in a range between $0.15 \mathrm{~dB}$ and $10.02 \mathrm{~dB}$. Generally speaking, the differences with respect to the static case are greater for the wider Doppler spectral characteristics.

However, the power spectral density for the highest and lowest frequencies should be also taken into account, as it represents the nonzero Doppler frequency contribution with respect to the zero component power density. To do so, the mean value of the fitting parameters mean $\{c, g\}$, which gives

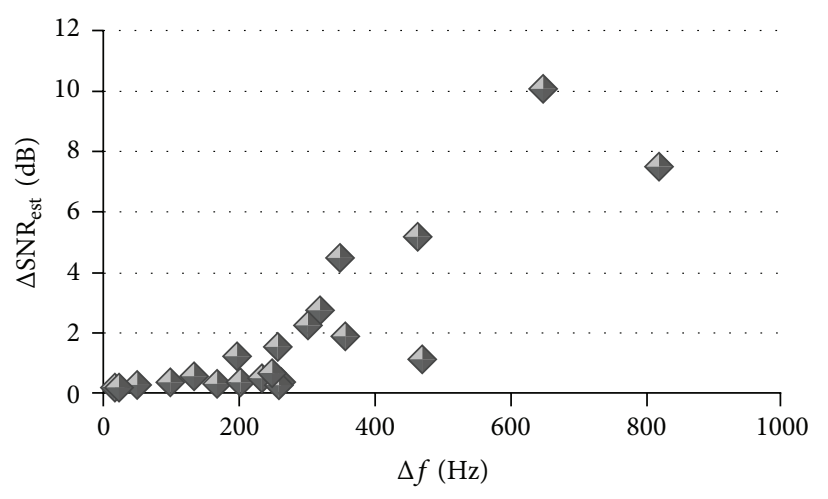

FIGURE 7: Difference in SNR values for BER equal to 2 . $10^{-4}\left(\Delta \mathrm{SNR}_{\mathrm{est}}\right)$ as a function of the spectral width $\Delta f$.

an idea of the power density for the highest and lowest frequencies, is calculated.

For instance, Example A (see Table 1, Figure 9, and Table 4 in Appendix), whose spectral width is $\Delta f_{\mathrm{A}}=$ $651.11 \mathrm{~Hz}$, shows a difference with respect to the static case of $\Delta \mathrm{SNR}_{\text {est_A }}=10.02 \mathrm{~dB}$, which is greater than the difference corresponding to Example $\mathrm{B}\left(\Delta \mathrm{SNR}_{\text {est_B }}=7.46 \mathrm{~dB}\right)$, despite the latter being wider $\left(\Delta f_{\mathrm{B}}=821.82 \mathrm{~Hz}\right)$. If we observe the values in Table 1, it can be concluded that Example A is more demanding in terms of SNR due to its lower mean $\{c, g\}$ value, which corresponds to stronger Doppler components at the edge, even when its spectral width is lower.

This effect is also noticeable when Examples $\mathrm{C}$ and $\mathrm{F}$ are examined. These two Doppler PSDs have almost equal spectral widths $\left(\Delta f_{\mathrm{C}}=468.29 \mathrm{~Hz}\right.$ and $\left.\Delta f_{\mathrm{F}}=473.87 \mathrm{~Hz}\right)$. However, the difference with respect to the static case is significantly higher for Example $\mathrm{C}$ due to its higher power spectral density for the highest frequencies (lower mean value of $\{c, g\}$ ), as observed in Table 1 .

For a graphical representation of this issue, data from Figure 7 are depicted in Figure 8 according to their mean $\{c, g\}$ values: green dots for mean $\{c, g\} \leq 35$, and blue dots for mean $\{c, g\}>35$. It can be observed that the differences with respect to the static case $\Delta \mathrm{SNR}_{\text {est }}$ are greater for the higher power spectral density values in the end frequencies, meaning stronger Doppler components at the edge. Considering the combined effect of the spectral width $(\Delta f)$ and the power spectral densities for the end frequencies 


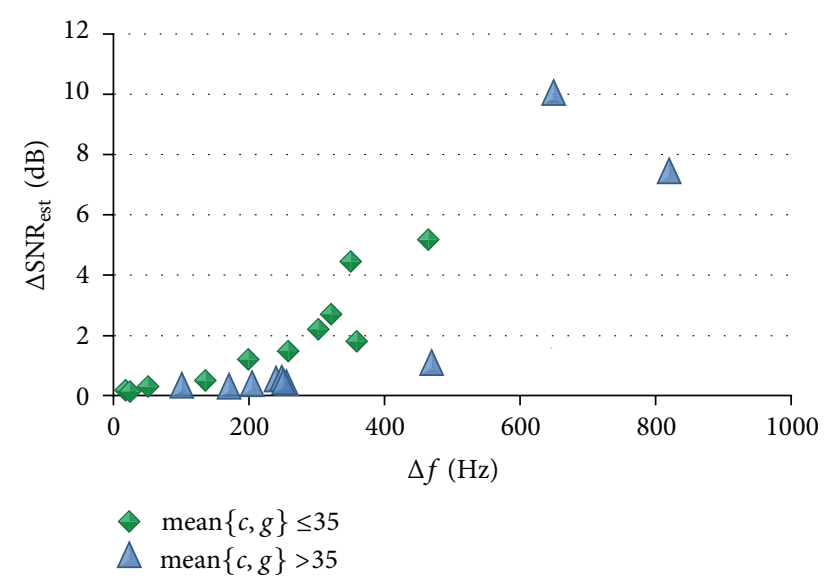

FIgURE 8: Difference in SNR values for BER equal to 2 . $10^{-4}\left(\Delta \mathrm{SNR}_{\mathrm{est}}\right)$ as a function of the spectral width $\Delta f$ and mean $\{c, g\}$.

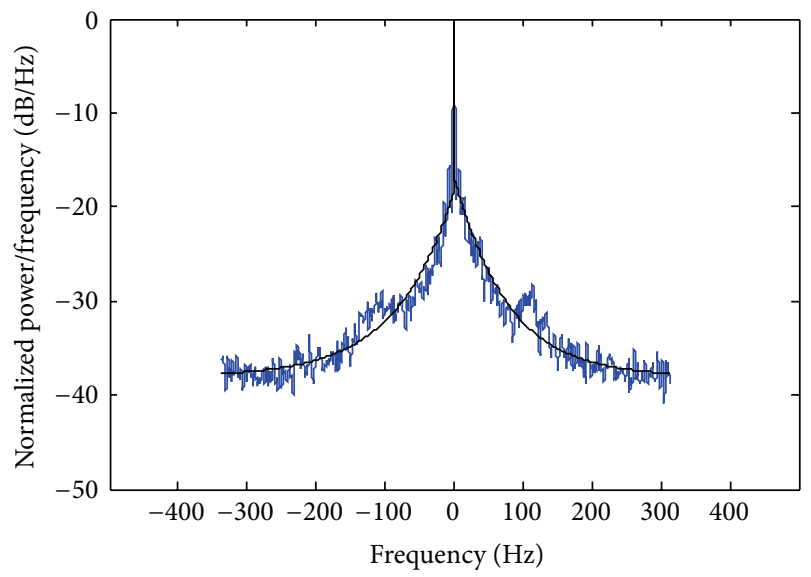

FIGURE 9: Example A: estimated PSD and corresponding exponential fitting $\left(a=19.72, b=1.22 \cdot 10^{-2}, c=38.05, d=21.42\right.$, $e=1.29 \cdot 10^{-2}, g=38.08, \Delta f=651.11$, and $\left.\gamma=0.04\right)$.

(mean $\{c, g\}$ ), the obtained results for the 24 selected Doppler spectra can be classified according to Table 2, providing a simple rule of thumb. It should be noted that the spectral widths are given as a function of the maximum Doppler frequency shift $f_{d}$.

It can be observed that all the Doppler spectra with spectral widths greater than $\Delta f \geq 3 / 4 \cdot f_{d}=330.48 \mathrm{~Hz}$ show differences with respect to the static case $\Delta \mathrm{SNR}_{\text {est }}>1 \mathrm{~dB}$. In a similar way, all the Doppler spectra with spectral widths lower than $\Delta f<1 / 2 \cdot f_{d}=220.32 \mathrm{~Hz}$ do not differ from the static case $\left(\Delta \mathrm{SNR}_{\text {est }}<1 \mathrm{~dB}\right)$. Between these two values, the differences with respect to the static case will depend on the power spectral density for the end frequencies.

Therefore, it can be concluded that the spectral width is a key parameter in order to determine potential degradations on OFDM systems, together with the value of the power spectral density for the highest and lowest frequencies.

4.2.2. Effect of the Asymmetry. The Doppler spectra with the highest grade of asymmetry (highest values of the parameter
TABLE 2: Relations between the spectral width, the PSD for the end frequencies, and the SNR difference with respect to the static case.

\begin{tabular}{lcc}
\hline$\Delta f(\mathrm{~Hz})$ & mean $\{c, g\}$ & $\Delta \mathrm{SNR}_{\mathrm{est}}(\mathrm{dB})$ \\
\hline$\Delta f \geq 3 / 4 \cdot f_{d}$ & any & $>1 \mathrm{~dB}$ \\
$1 / 2 \cdot f_{d} \geq \Delta f>3 / 4 \cdot f_{d}$ & $\leq 35$ & $>1 \mathrm{~dB}$ \\
$\Delta f<1 / 2 \cdot f_{d}$ & $>35$ & $<1 \mathrm{~dB}$ \\
\hline
\end{tabular}

$\gamma$ ) do not seem to cause further degradation with respect to the degradation corresponding to that spectral width and PSD values for the end frequencies. For instance, in the case of Examples D, E, and G, the three of them have similar spectral widths and power spectral densities for the highest and lowest frequencies, and thus, their SNR differences with respect to the static case $\Delta \mathrm{SNR}_{\text {est }}$ are similar despite their different degrees of asymmetry (see Tables 1 and 4 in Appendix).

4.2.3. Goodness of Fit. Until now, only the results corresponding to the BER versus SNR curves due to the estimated Doppler spectra have been analyzed. However, it is also important to validate the goodness of the proposed exponential fitting, and check the differences in the BER versus SNR curves corresponding to the estimated Doppler spectra and their exponential fittings. To do so, the BER value equal to $2 \cdot 10^{-4}$ is taken as a reference, and the difference between the curves corresponding to the empirical and the exponential PSDs is calculated and expressed as $\triangle \mathrm{SNR}_{\text {exp }}$.

From the selected examples, the results of the BER versus SNR curves show that the curves of the exponential fittings with coefficient of determination $R^{2} \geq 0.8$ are practically coincident with the curves of the empirical Doppler spectra. It should be remarked that, as previously commented, the mean $R^{2}$ value of the whole empirical data set is 0.87 .

More precisely, the difference between the empirical and the fitted curves depends on the additional degradation with respect to the static case due to the empirical Doppler PSD. That is to say, for all the Doppler PSDs whose empirical curves are practically coincident with the static curve, their exponential fitting curves are also coincident even if $R^{2}<0.8$.

4.2.4. Overview of the Case under Study. It should be noted that the aim of the 24 Doppler spectra used for simulation was to look for critical cases that allowed a thorough study of the potential degradation due to the Doppler PSDs and its connection with the previously commented parameters.

However, these cases of potential degradation are few within the whole available data set. Applying the conclusions from the previous subsections, an estimation of the percentage of cases from the case under study which will be more affecting to OFDM signals can be obtained.

To do so, the Doppler effect is considered to be noticeable when the SNR with respect to static case is $\Delta \mathrm{SNR}_{\text {est }}>1 \mathrm{~dB}$; on the contrary, differences with respect to the static case $\Delta \mathrm{SNR}_{\text {est }}<1 \mathrm{~dB}$ are considered to be negligible. With respect to the goodness of fit, differences between the empirical and the fitted curves are considered to be acceptable if 


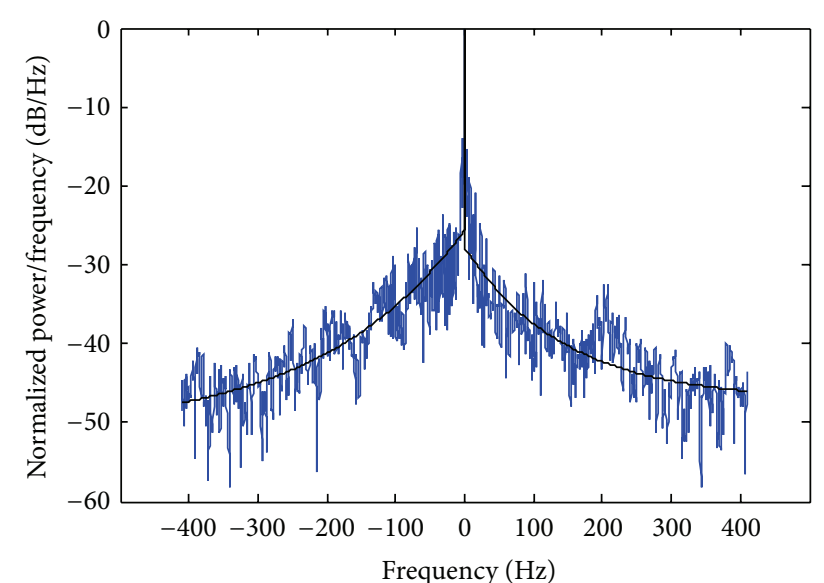

FIGURE 10: Example B: estimated PSD and corresponding exponential fitting $\left(a=25.50, b=4.73 \cdot 10^{-2}, c=51.17, d=19.40\right.$, $e=6.87 \cdot 10^{-2}, g=47.15, \Delta f=821.82$, and $\left.\gamma=0.00\right)$.

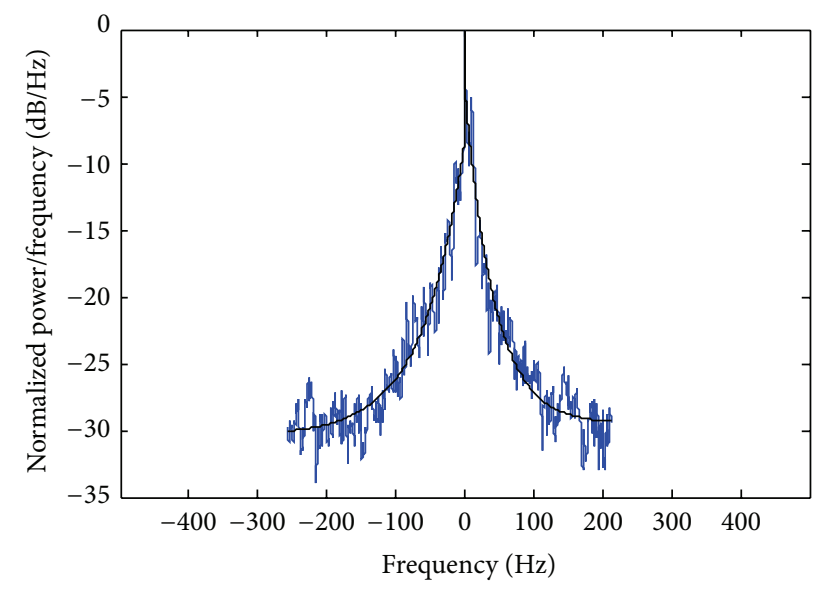

FIGURE 11: Example C: estimated PSD and corresponding exponential fitting $\left(a=21.98, b=1.65 \cdot 10^{-2}, c=30.39, d=25.09\right.$, $e=2.33 \cdot 10^{-2}, g=29.53, \Delta f=468.29$, and $\gamma=0.09$ ).

$\Delta \mathrm{SNR}_{\text {exp }} \leq 0.5 \mathrm{~dB}$. According to this, the percentages of measured Doppler spectra according to their potential degradation and goodness of fit are given in Table 3.

According to the obtained results, the kind of time variability encountered for a majority of cases will not cause further degradation on OFDM systems with respect to the same propagation channel in static conditions. However, these potentially degrading situations will affect the reception thresholds of digital communication systems and should be taken into account for planning purposes. Apart from that, the proposed exponential model fits the empirical data for a vast majority of cases.

\section{Conclusion}

This paper presents the Doppler characterization of the signals scattered by the wind turbines in the UHF band for near field condition in the context of signal scattering. The study

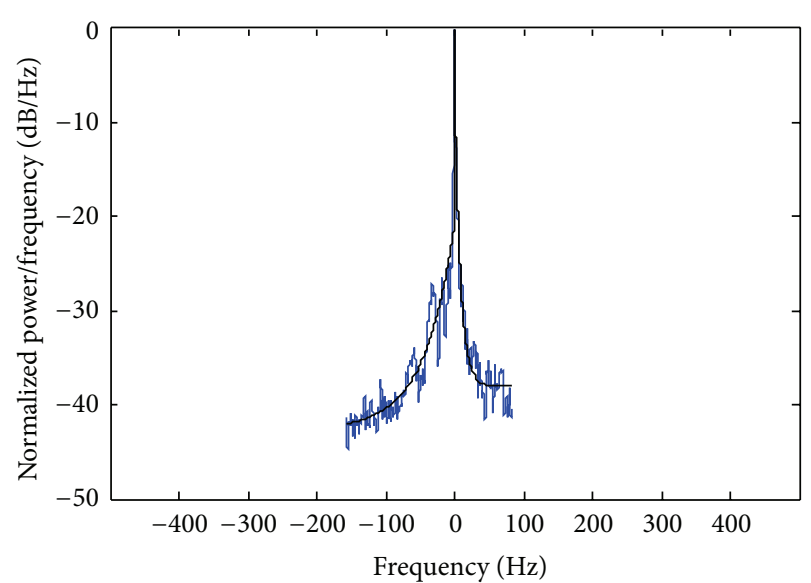

FIGURE 12: Example D: estimated PSD and corresponding exponential fitting $\left(a=21.72, b=2.29 \cdot 10^{-2}, c=42.64, d=31.74\right.$, $e=1.16 \cdot 10^{-2}, g=37.99, \Delta f=241.12$, and $\gamma=0.31$ ).

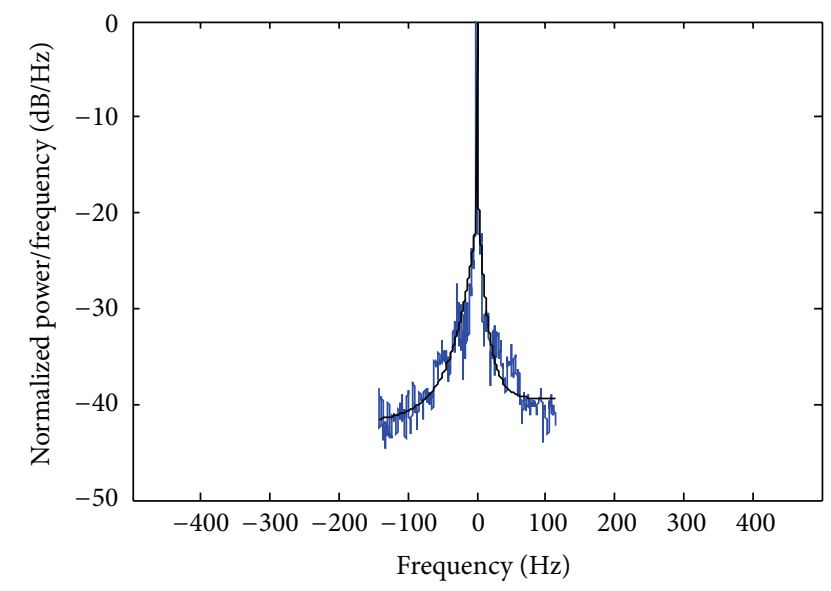

FIGURE 13: Example E: estimated PSD and corresponding exponential fitting $\left(a=21.36, b=2.94 \cdot 10^{-2}, c=41.87, d=24.45\right.$, $e=6.81 \cdot 10^{-2}, g=39.45, \Delta f=256.45$, and $\gamma=0.10$ ).

TABLE 3: Percentage of the total number of psds according to their degree of degradation due to Doppler effect and goodness of the exponential fit.

\begin{tabular}{llc}
\hline $\begin{array}{l}\text { Degradation due to Doppler effect } \\
\left(\triangle \text { SNR }_{\text {est }}\right)\end{array}$ & $\begin{array}{l}\text { To be } \\
\text { considered }\end{array}$ & $9 \%$ \\
& Negligible & $91 \%$ \\
\hline \multirow{2}{*}{ Goodness of fit } & Acceptable & $95 \%$ \\
& Unacceptable & $5 \%$ \\
\hline
\end{tabular}

is based on empirical data obtained in the surroundings of a real wind farm in Spain. To do so, the Welch's method with the adaptation of its parameters to the particular conditions of each signal has proved to be suitable for obtaining the Doppler PSDs.

Then, after an evaluation of the existing Doppler spectrum models, it is concluded that none of them applies to the special mobility features of the propagation channel in 
TABLE 4: Fitting parameters of the representative Doppler spectra.

\begin{tabular}{|c|c|c|c|c|c|c|c|c|}
\hline Example & $a$ & $b$ & $c$ & $d$ & $e$ & $g$ & $f_{\min }$ & $f_{\max }$ \\
\hline A & 19.72 & $1.22 \cdot 10^{-2}$ & 38.05 & 21.42 & $1.29 \cdot 10^{-2}$ & 38.08 & -337.25 & 313.87 \\
\hline B & 25.50 & $4.73 \cdot 10^{-2}$ & 51.17 & 19.40 & $6.87 \cdot 10^{-2}$ & 47.15 & -410.91 & 410.91 \\
\hline $\mathrm{C}$ & 21.98 & $1.65 \cdot 10^{-2}$ & 30.39 & 25.09 & $2.33 \cdot 10^{-2}$ & 29.53 & -256.20 & 212.09 \\
\hline $\mathrm{D}$ & 21.72 & $2.29 \cdot 10^{-2}$ & 42.64 & 31.74 & $1.16 \cdot 10^{-2}$ & 37.99 & -158.37 & 82.76 \\
\hline $\mathrm{E}$ & 21.36 & $2.94 \cdot 10^{-2}$ & 41.87 & 24.45 & $6.81 \cdot 10^{-2}$ & 39.45 & -141.61 & 114.85 \\
\hline $\mathrm{F}$ & 27.01 & $1.82 \cdot 10^{-2}$ & 44.62 & 25.49 & $1.71 \cdot 10^{-2}$ & 44.58 & -230.52 & 243.35 \\
\hline $\mathrm{G}$ & 21.21 & $3.30 \cdot 10^{-2}$ & 40.20 & 24.65 & $2.85 \cdot 10^{-2}$ & 40.74 & -116.82 & 131.14 \\
\hline
\end{tabular}

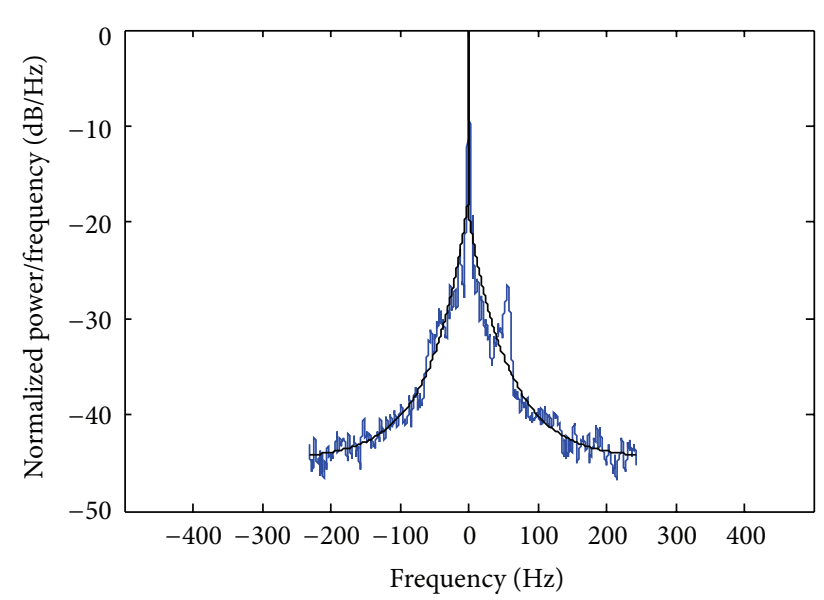

FIGURE 14: Example F: estimated PSD and corresponding exponential fitting $\left(a=27.01, b=1.82 \cdot 10^{-2}, c=44.62, d=25.49\right.$, $e=1.71 \cdot 10^{-2}, g=44.58, \Delta f=473.87$, and $\left.\gamma=0.03\right)$.

presence of a wind farm. Therefore, a new exponential model has been proposed. This model is composed of a Dirac delta for the zero Doppler frequency, which corresponds to the static supporting mast of the wind turbine and results in the component of maximum amplitude and side components of decreasing power spectral density for the lowest and highest frequencies, which correspond to the movement of the blades.

This exponential model allows, on a first approach, to limit the Doppler spectra in frequency and filter the noise floor. Then, on a second iteration, this exponential model allows the characterization of the Doppler spectra after the frequency limiting.

In order to analyze the most influential characteristics of the Doppler spectra on OFDM signal degradation, BER versus SNR curves of some representative examples have been carried out. The main conclusion is that one of the most critical parameters is the spectral width, along with the power spectral density values for the end frequencies. The goodness of fit of the proposed exponential fitting has also been proved, because both the empirical spectra and the exponential approximations provide similar BER versus SNR curves for a vast majority of cases.

Finally, it should be noted that the time variability of the scattered signals does not seem to cause further degradation on OFDM signals for a high percentage of cases. Although the

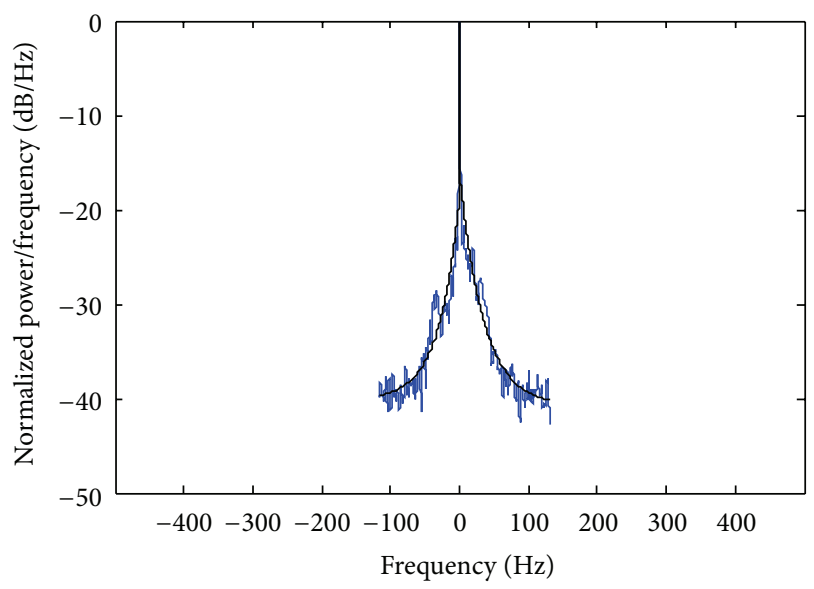

FIGURE 15: Example G: estimated PSD and corresponding exponential fitting $\left(a=21.21, b=3.30 \cdot 10^{-2}, c=40.20, d=24.65\right.$, $e=2.85 \cdot 10^{-2}, g=40.74, \Delta f=247.96$, and $\left.\gamma=0.06\right)$.

probability of time variability causing reception problems is low due to the robustness of digital systems, it should be taken into account in order to estimate the corresponding reception thresholds. To do so, the representative examples of Doppler spectra given by the fitting parameters included in Table 4 (Appendix) can be used to estimate new situations of time variability due to the rotating blades of wind turbines. First, these Doppler spectra should be normalized as a function of their corresponding maximum bistatic Doppler frequency shift $f_{\mathrm{B}_{-} \max }$. This parameter is a function of the wind turbine characteristics (blade length $l$ and maximum rotation rate $w_{\max }$ ), relative position transmitter-wind turbine-receiver (bistatic angle $\beta$ ), and working frequencies (wavelength $\lambda$ ). Thus, in order to extend the applicability of the results, the maximum bistatic Doppler frequency shift $f_{\mathrm{B}_{-} \max }$ corresponding to the specific characteristics of the new case under study should be calculated according to (10), and the provided Doppler spectra adapted accordingly.

\section{Appendix}

See Table 4 and Figures 9, 10, 11, 12, 13, 14, and 15.

\section{Acknowledgments}

The authors would like to thank the partners from Iberdrola Renovables for their continuous support and involvement in 
the study of the wind turbine effects on the radiocommunication services. Special thanks also to Itelazpi and Abertis Telecom for their kind collaboration in this work. This work has been supported in part by the European Union FP7 (Grant Agreement no. 296164), by the Spanish Ministry of Economy and Competitiveness (projects TEC2012-32370 and TEC2009-14201), and by the Basque Government (GIC 07/ 110-IT-374-07, SAIOTEK program and program for the training of the researcher staff BFI08.230).

\section{References}

[1] I. Angulo, D. de la Vega, N. Cau et al., "Empirical evaluation of the impact of wind turbines on DVB-T reception quality," IEEE Transactions on Broadcasting, vol. 58, no. 1, pp. 1-9, 2012.

[2] I. Angulo, D. de la Vega, C. Fernandez et al., "An empirical comparative study of prediction methods for estimating multipath due to signal scattering from wind turbines on digital TV services," IEEE Transactions on Broadcasting, vol. 57, no. 2, pp. 195203, 2011.

[3] I. Angulo, D. de la Vega, O. Grande et al., "Methodology for the empirical analysis of the scattering signals from a wind turbine," in Proceedings of the Loughborough Antennas and Propagation Conference (LAPC'09), pp. 553-556, Loughborough, UK, November 2009.

[4] D. de la Vega, I. Orcajo, I. Armentia et al., "Use of the channel impulse response of the DVB-T service for the evaluation of the reflected signals of wind farms," in Proceedings of the IEEE International Instrumentation and Measurement Technology Conference (IMTC '08), Victoria, Canada, May 2008.

[5] E. F. Knott, J. F. Shaeffer, and M. T. Tuley, Radar Cross Section: Its Prediction, Measurement and Reduction, Artech House, Norwood, Mass, USA, 1985.

[6] N. Whitelonis, S. Yang, and H. Ling, "Application of near-field to far-field transformation to doppler features from wind turbine scattering," IEEE Transactions on Antennas and Propagation, vol. 60, no. 3, pp. 1660-1665, 2012.

[7] J. G. Proakis and D. G. Manolakis, Digital Signal Processing: Principles, Algorithms, and Applications, Prentice Hall, Englewood Cliffs, NJ, USA, 1996.

[8] F. J. Harris, "On the use of windows for harmonic analysis with the discrete Fourier transform," Proceedings of the IEEE, vol. 66, no. 1, pp. 51-83, 1978.

[9] A. V. Oppenheim, A. S. Willsky, and I. T. Young, Signals and Systems, Prentice Hall, Englewood Cliffs, NJ, USA, 1983.

[10] P. Stoica and R. Moses, Introduction to Spectral Analysis, Prentice Hall, Upper Saddle River, NJ, USA, 1997.

[11] S. L. Marple, Digital Spectral Analysis, Prentice Hall, Upper Saddle River, NJ, USA, 1987.

[12] A. Schuster, "On the investigation of hidden periodicities," Terrestrial Magnetism, vol. 3, pp. 13-41, 1898.

[13] M. S. Bartlett, "Smoothing periodograms from time-series with continuous spectra," Nature, vol. 161, no. 4096, pp. 686-687, 1948.

[14] P. Welch, "The use of fast Fourier transform for the estimation of power spectra: a method based on time averaging over short, modified periodograms," IEEE Transactions on Audio and Electroacoustics, vol. 15, no. 2, pp. 70-73, 1967.

[15] G. J. Poupart, "Wind farms impact on radar aviation interestsfinal report," FES W/14/00614/00/REP, DTI PUB URN 03/1294, QinetiQ, 2003.
[16] A. Naqvi, S. T. Yang, and H. Ling, "Investigation of doppler features from wind turbine scattering," IEEE Antennas and Wireless Propagation Letters, vol. 9, pp. 485-488, 2010.

[17] A. Naqvi, Investigation of Doppler features resulting from wind turbine scattering [M.S. thesis], The University of Texas at Austin, 2010.

[18] B. Gallardo-Hernando, F. Pérez-Martínez, and F. AguadoEncabo, "Statistical characterization of wind turbine clutter in C-band radars," in Proceedings of the International Conference on Radar, pp. 360-364, September 2008.

[19] M. Patzold, Mobile Fading Channels, John Wiley \& Sons, New York, NY, USA, 2002.

[20] W. C. Jakes, Microwave Mobile Channels, John Wiley \& Sons, New York, NY, USA, 1974.

[21] R. H. Clarke and W. L. Khoo, "3-D mobile radio channel statistics," IEEE Transactions on Vehicular Technology, vol. 46, no. 3, pp. 798-799, 1997.

[22] D. C. Cox, "Delay Doppler characteristics of multipath propagation at $910 \mathrm{MHz}$ in a suburban mobile radio environment," IEEE Transactions on Antennas and Propagation, vol. 20, no. 5, pp. 625-635, 1972.

[23] IEEE 802.16 Broadband Wireless Access Working Group, "Channel models for fixed wireless applications," IEEE 802.16a03/01, 2003.

[24] M. Skolnik, Radar Handbook, McGraw-Hill, New York, NY, USA, 3rd edition, 2008.

[25] H. Hashemi, M. McGuire, T. Vlasschaert, and D. Tholl, "Measurements and modeling of temporal variations of the indoor radio propagation channel," IEEE Transactions on Vehicular Technology, vol. 43, no. 3, pp. 733-737, 1994.

[26] A. Domazetovic, L. J. Greenstein, N. B. Mandayam, and I. Seskar, "Estimating the Doppler spectrum of a short-range fixed wireless channel," IEEE Communications Letters, vol. 7, no. 5, pp. 227-229, 2003.

[27] V. Erceg, L. Schumacher, P. Kyritsi et al., "TGn Channel Models," Tech. Rep. IEEE P802.11 Wireless LANs, 2004.

[28] W. Yamada, K. Nishimori, Y. Takatori, and Y. Asai, "Statistical analysis and characterization of doppler spectrum in large office environment," in Proceedings of the International Symposium on Antennas and Propagation (ISAP '09), pp. 564-567, Bangkok, Thailand, 2009.

[29] D. S. Baum, D. Gore, R. Nabar et al., "Measurement and characterization of broadband MIMO fixed wireless channels at $2.5 \mathrm{GHz}$," in Proceedings of the IEEE International Conference on Personal Wireless Communications, pp. 203-206, December 2000.

[30] I. Angulo, D. de la Vega, O. Rodríguez, O. Grande, D. Guerra, and P. Angueira, "Analysis of the mast contribution to the scattering pattern of wind turbines in the UHF band," in Proceedings of the 5th European Conference on Antennas and Propagation (EUCAP '11), pp. 707-711, April 2011.

[31] R. G. D. Steel and J. H. Torrie, Principles and Procedures of Statistics, McGraw-Hill, New York, NY, USA, 1960.

[32] J. Montalbán, M. M. Velez, G. Prieto, I. Eizmendi, G. BerjonEriz, and J. L. Ordiales, "On approaching to generic channel equalization techniques for OFDM based systems in time variant channels," in Proceedings of the IEEE International Symposium Broadband Multimedia Systems and Broadcasting (BMSB '11), pp. 1-6, June 2011.

[33] ETSI, "Digital Video Broadcasting (DVB), framing structure, channel coding and modulation for digital terrestrial television," ETSI EN 300744 V1.6.1, 2009. 
[34] M. C. Jeruchim, P. Balaban, and K. S. Shanmugan, Simulation of Communication Systems: Modeling, Methodology, and Techniques, Plenum Press; Kluwer Academic, New York, NY, USA, 2nd edition, 2000.

[35] J. D. Parsons, The Mobile Radio Propagation Channel, Halsted Press, 1992. 

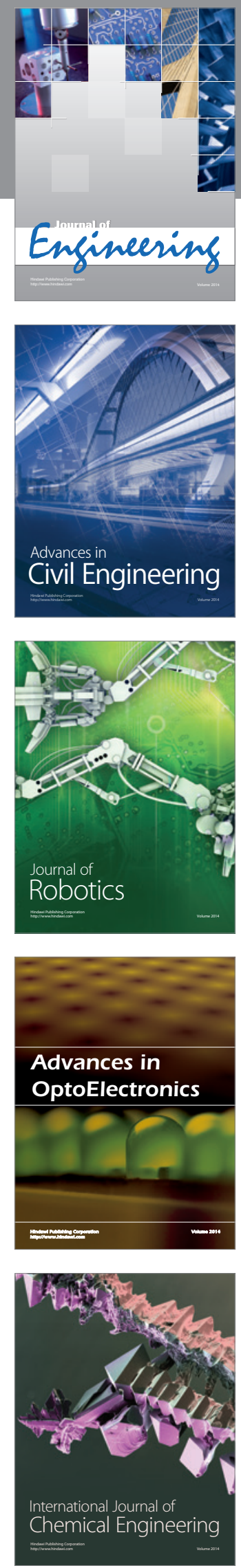

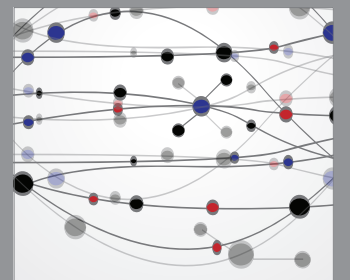

The Scientific World Journal
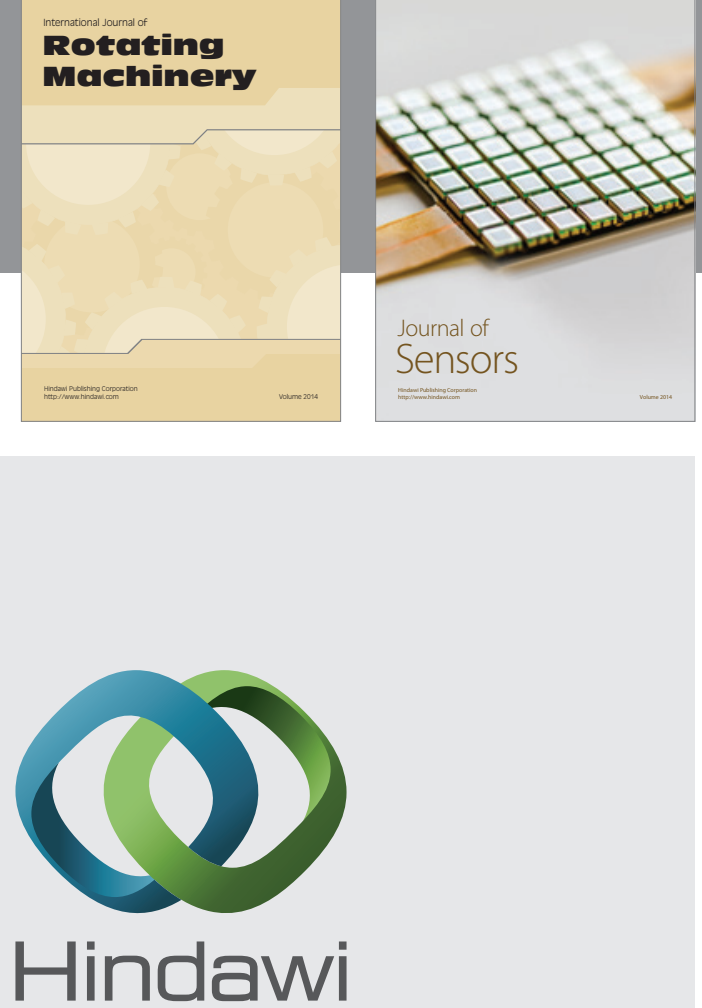

Submit your manuscripts at http://www.hindawi.com
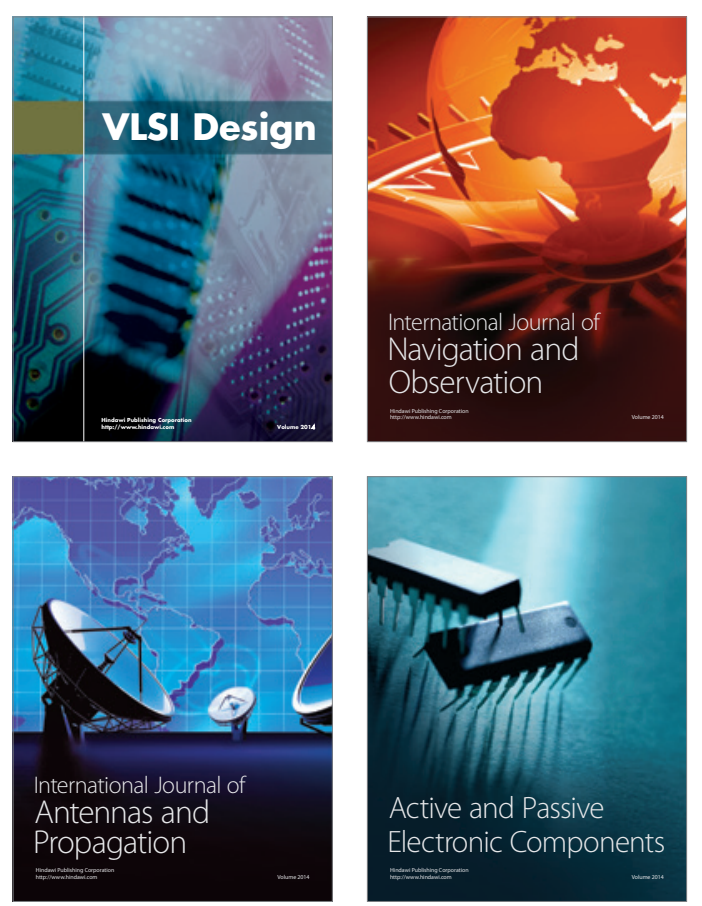
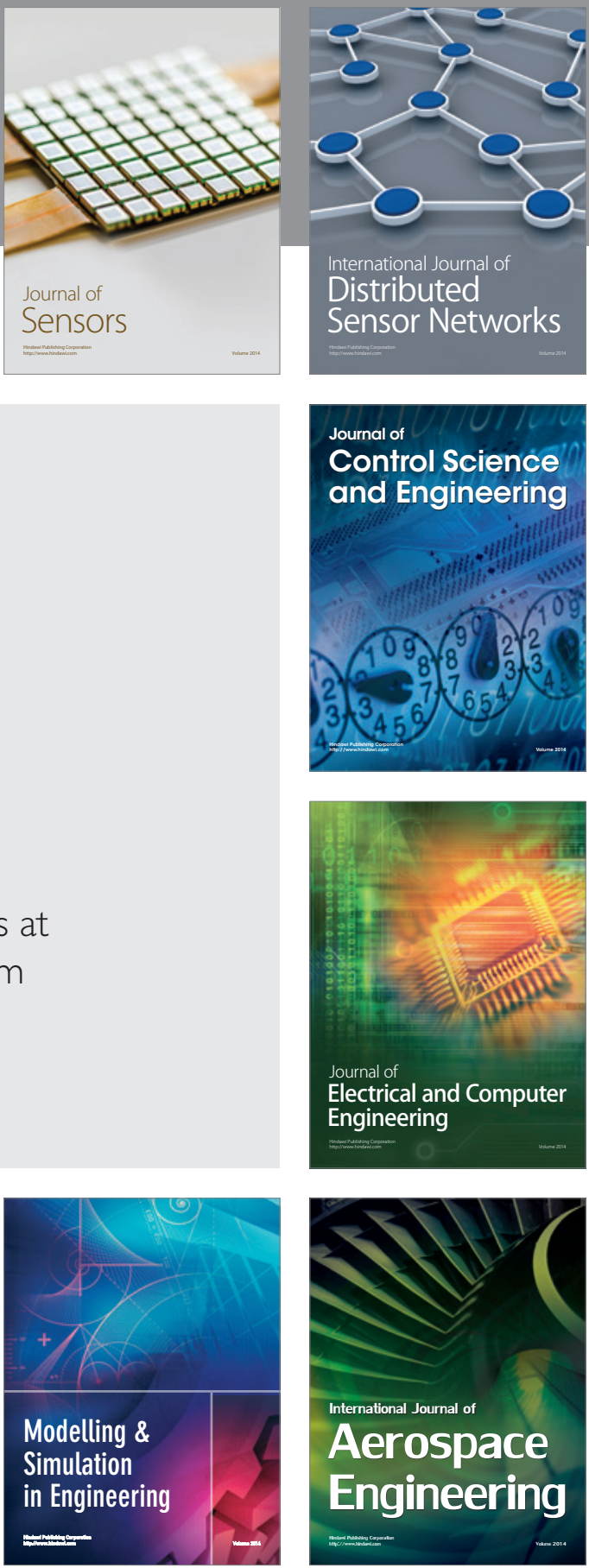

Journal of

Control Science

and Engineering
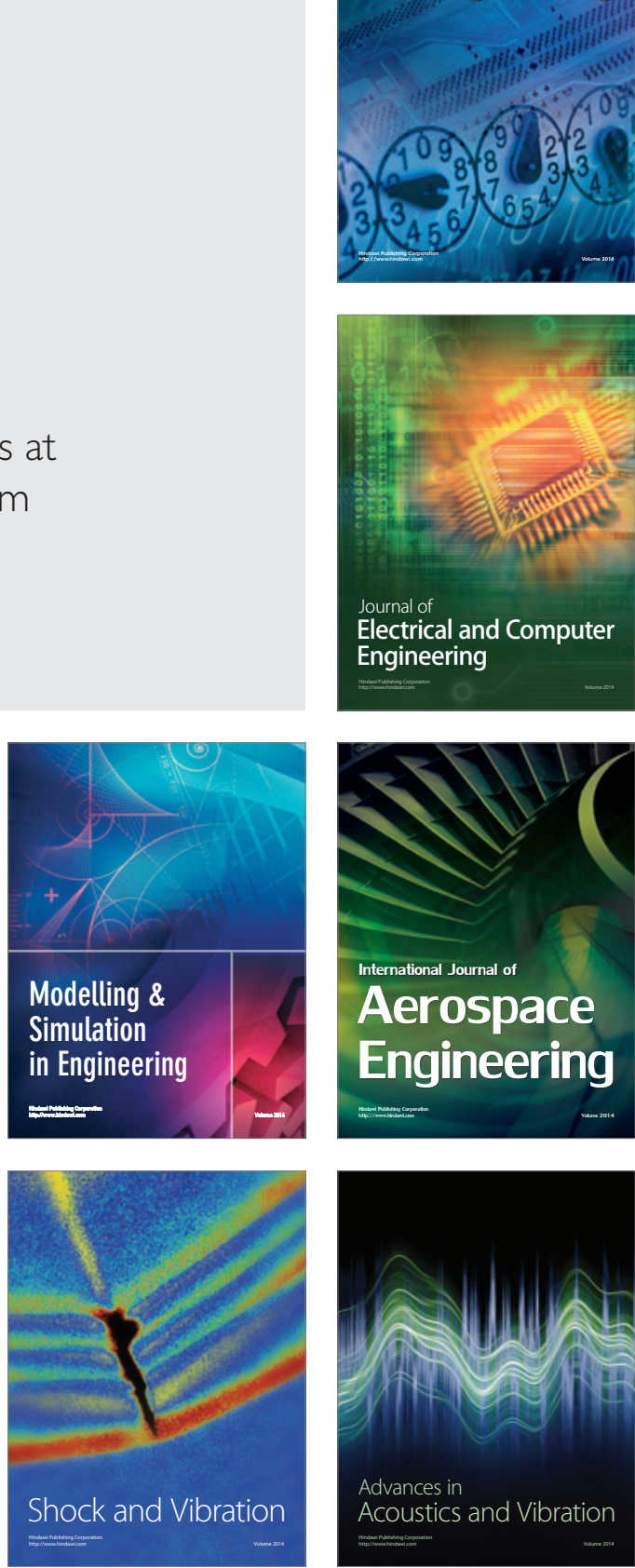